\title{
La concentración productiva como determinante de la oferta de crédito en América Latina
}

\author{
The productive concentration as a determinant \\ for credit offer in Latin America
}

A concentração produtiva como determinante da oferta de crédito na América Latina

\author{
Nicolás Barriga \\ Universidad de las Américas (Quito, Ecuador) \\ nicolas.barriga@outlook.com
}

DOI: https://doi.org/10.32719/25506641.2019.6.7

Fecha de presentación: 8 de junio de 2019 • Fecha de aceptación: 15 de agosto de 2019 Artículo de investigación

Licencia Creative Commons

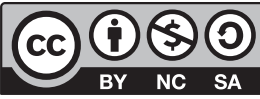




\section{Nicolás Barriga}

\section{Resumen}

El crédito, en los países latinoamericanos, se emplea como un mecanismo de financiamiento de nuevos emprendimientos, empresas e industrias, permitiendo el desarrollo de encadenamientos productivos en las economías. A su vez, la región latinoamericana se ha caracterizado por mantener una producción concentrada en determinados sectores económicos. Por consiguiente, esta investigación tiene como objetivo determinar la relación existente entre la concentración productiva -medida a través del índice Herfindahl-Hirschman- y la aceleración de la oferta de crédito en América Latina. Para alcanzar este fin, se empleó un modelo econométrico de datos de panel para quince economías representativas de la región, en el período 2004-2016. Los principales resultados de la investigación indican que la concentración productiva de las economías latinoamericanas desacelera la oferta de crédito en la región.

Palabras clave: Oferta de crédito, concentración productiva, índice Herfindahl-Hirschman.

JEL: D24 Producción; productividad del capital y del total de factores; capacidad.

\section{Abstract}

Credit, in Latin American countries, is used as a financing mechanism for new ventures, companies and industries, allowing the development of productive chains in the economy. At the same time, Latin American region has been characterized by maintaining a concentrated production in certain economic sectors. Therefore, this research aims to determine the relationship between productive concentration -measured through the Herfindahl-Hirschman index - and the acceleration of credit offer in Latin America. In order to achieve this goal, an econometric model of panel data was used for fifteen representative economies in the region, in the period 2004-2016. The main results of this research indicate that the productive concentration of Latin American economies slows the credit offer in this region.

Keywords: Credit offer, productive concentration, Herfindahl-Hirschman index.

JEL: D24: Production; productivity of capital and all factors; capacity.

\section{Resumo}

O crédito, nos países latino-americanos, se emprega como um mecanismo de financiamento de novos empreendimentos, empresas e indústrias, permitindo o desenvolvimento de encadeamentos produtivos nas economias. Por sua vez, a região latino-americana foi caracterizada por manter uma produção concentrada em determinados setores econômicos. Portanto, esta investigação tem como objetivo determinar a relação existente entre a concentração produtiva - medida através do índice Herfindahl-Hirschman-e a aceleração da oferta de crédito na América Latina. Para alcançar este fim, se empregou um modelo econométrico de dados de painel para quinze economias representativas da região, no período 2004-2016. Os principais resultados da investigação indicam que a concentração produtiva das economias latino-americanas desacelera a oferta de crédito na região.

Palavras-chave: Oferta de crédito, concertação produtiva, índice Herfindahl-Hirschman.

JEL: D24: Produção; produtividade do capital e do total de fatores; capacidade. 


\section{Introducción}

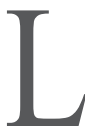

a estructura productiva de la región latinoamericana presenta, históricamente, una concentración en sectores económicos que generan un escaso valor agregado en los bienes producidos. De acuerdo con Pérez (2017), en presencia de centralización productiva, se entorpece la creación de nuevas empresas o industrias que permitan viabilizar los engranajes y encadenamientos productivos. Esta concentración productiva repercute en el sistema financiero, pues los bancos destinan los créditos a los sectores más representativos de la economía, generándose un riesgo conocido como concentración sectorial de créditos (Hibbeln 2010). Para gestionar y minimizar el riesgo por concentración, las instituciones bancarias realizan acciones que impactan en el otorgamiento de créditos (Düllmann 2006).

El propósito de esta investigación es determinar si la concentración productiva afecta al volumen de crédito en América Latina. Se tomó como muestra a nueve países sudamericanos (Argentina, Bolivia, Brasil, Chile, Colombia, Ecuador, Paraguay, Uruguay y Perú), cinco países de Centroamérica (Costa Rica, Guatemala, Panamá, Honduras y Nicaragua), y uno de Norteamérica (México), durante el período 2004-2016. Para establecer la relación entre volumen de crédito y concentración productiva se empleó un modelo de panel de datos con frecuencia anual, donde la variable dependiente es el volumen de crédito real y las variables de control son el PIB, depósitos a la vista, tasa de interés y precio del petróleo, expresadas en términos reales, y una variable de concentración, calculada mediante el índice Herfindahl-Hirschman.

La investigación está organizada en cuatro secciones. En la primera sección se plantean las discusiones teóricas que respaldan la relación entre la estructura productiva y las decisiones de colocación de créditos a partir de la gestión de intermediación que realizan los bancos. La sección siguiente corresponde al contexto, en la que se evidencia el comportamiento del cré- 
dito y la estructura productiva de la región latinoamericana en el período 2004-2016. La sección tercera corresponde a la metodología y resultados, en donde se expone el modelo econométrico empleado para demostrar el impacto de la estructura productiva en el otorgamiento de crédito en la región. Por último, se presenta el apartado de conclusiones y recomendaciones que se obtuvieron de los resultados de la investigación.

\section{Marco teórico}

\section{El sistema financiero y los bancos}

El sistema financiero es el conjunto de entidades que, a través de mercados financieros, tienen como función la generación, captación y dirección del ahorro de una economía proveniente de los agentes superavitarios hacia los agentes deficitarios (Freixas y Rochet 1999). Este proceso es realizado por intermediarios financieros, tanto públicos como privados, encargados de facilitar la transferencia eficiente de los recursos, los cuales se clasifican en cooperativas, mutualistas, cajas de ahorro y bancos (Rodríguez 1996). De acuerdo con Mishkin (2007), los bancos son los intermediarios financieros que mejor distribuyen los recursos entre los agentes gracias a sus economías de escala, cumpliendo con funciones específicas.

Para el cumplimiento del rol de intermediación que adoptan los bancos, estos deben especializarse en algunas actividades que facilitan este proceso de asignación y captación de recursos. Acorde con la sistematización realizada por Levine (1997), las especializaciones de los bancos son: gestión del riesgo, recopilación de información; reducción de costos de agencia; y transformación de activos.

Respecto a la primera especialización, la gestión del riesgo se refiere a las acciones que los bancos realizan para minimizar el riesgo proveniente de sus operaciones, a través de la creación de provisiones o diversificación de la cartera (Bencivenga y Smith 1991). El cumplimiento de esta función permite a los bancos mejorar su manejo administrativo al obtener mayores retornos y evitar problemas financieros en las economías (Audretsch, Bönte y Prashanth 2009). La segunda especialización se refiere a la reducción de 
asimetrías de información entre prestamistas y prestatarios, debido a que los agentes no siempre revelan toda la información (Akerlof 1970), los acreedores no distinguen a los demandantes de crédito que presentan una mayor probabilidad de impago. Por lo tanto, la ejecución de esta función reduce el riesgo por incumplimiento de créditos y permite una canalización eficiente de los recursos desde el sistema financiero hacia los sectores productivos (Levine 1997). La tercera función, la reducción de costos de agencia, se refiere a la minimización de los costos necesarios en supervisión que los bancos realizan para asegurarse el cumplimiento de las obligaciones adquiridas por los prestatarios (Rajan y Zingales 1998). Finalmente, la transformación de activos es la capacidad bancaria que transforma los ahorros de los agentes superavitarios en recursos que financian a los agentes deficitarios (Sirri y Tufano 1995). Esta transformación de activos financieros, realizada por los bancos, es en plazo, monto y riesgo (Catarineu y Pérez 2008). Esta competencia permite a los bancos asignar de manera eficiente los recursos, a través del crédito, hacia los agentes que realicen actividades de producción o consumo (Greenwood y Jovanic 1990). Además, este proceso vincula tanto a los prestamistas como a los prestatarios, a través de un mercado de crédito (Levine 1997). En ese sentido, el mercado de crédito funciona de la interacción entre demanda y oferta de crédito, donde la tasa de interés es el precio que equilibra este mercado (Wojnilower 1985).

Por un lado, la demanda de crédito se compone de agentes que desean mantener un consumo estable en el tiempo y empresas que desean incrementar sus niveles de inversión (Pazarbasioglu 2003). Dentro de los principales determinantes de la demanda de crédito se encuentran: la actividad económica, el nivel de ingresos, las expectativas y la tasa de interés (Mochón y Beker 1997). La actividad económica presenta una relación positiva con la demanda de crédito puesto que, según King y Plosser (2001), si una economía crece, la masa monetaria y los salarios aumentan, dando como resultado una mayor capacidad de endeudamiento por parte de los individuos. Además, existen actividades productivas que demandan elevados niveles de crédito, debido al capital de trabajo necesario (Rodríguez y Gómez 2002). El nivel de ingresos es un determinante que mantiene una relación positiva con la demanda de crédito en virtud de que, si un agente presenta mayores ingresos, su demanda de crédito aumenta para satisfacer el incremento en el consumo. Según Akerlof y Shiller (2009), las expectativas son determinantes de la demanda 
de crédito porque las decisiones de consumo o inversión por parte de los agentes económicos obedecen a olas de optimismo o pesimismo provenientes de la confianza e incertidumbre. Finalmente, la tasa de interés mantiene una relación inversa con la demanda de crédito, puesto que representa el costo de endeudamiento y también define la preferencia intertemporal de los individuos para consumir o ahorrar (Friedman 1957; Domínguez y Naranjo 2009).

Por otro lado, la oferta de crédito se compone de intermediarios financieros que invierten recursos en una economía con la finalidad de obtener la mejor combinación entre rentabilidad y riesgo (Pazarbasioglu 2003). Los recursos que manejan los intermediarios financieros, específicamente los bancos, provienen de los ahorros y depósitos del público. Estos recursos pueden ser prestados, a corto, mediano o largo plazo, a entidades públicas, privadas o particulares (Rodríguez 1996). Suárez (1992) definió a este proceso de colocación de recursos como oferta de crédito bancario. Según Alexander y Caramazza (1994), Suárez (1992); Hicks (1980) y Holub, Nyklíček y Pavel (2014) coinciden en que los determinantes de la oferta de crédito son: la actividad económica, los depósitos a la vista, las expectativas y la tasa de interés.

La actividad económica es determinante en la oferta de crédito dado que, si esta aumenta, se reduce la probabilidad de que los prestatarios incumplan con sus obligaciones y, como consecuencia, los bancos disminuyen las reservas para incrementar la colocación de créditos (Bernanke y Gertler 1986). Además, Blinder (1987) demostró que, cuando la actividad económica es favorable, los bancos incrementan su volumen de crédito como derivación del aumento de los depósitos por parte del público. Este proceso es posible, según King (1986), pues los depósitos a la vista son considerados por los agentes como una fuente de reserva de riqueza y por los bancos como la principal fuente de liquidez para aumentar la colocación de crédito. De esta manera, existe una relación directa entre los depósitos y el volumen de crédito (Murcia y Piñeros 2006). Respecto a las expectativas como determinante de la oferta de crédito, Hirshleifer y Yu (2012) aseguran que estas se forman de manera adaptativa, es decir, según la predicción derivada de una relación funcional dinámica, suponen que acontecimientos ocurridos en el pasado se cumplirán en el futuro. Los acreedores consideran que las expectativas les permiten crear predicciones acerca del desenvolvimiento de la economía en un período de tiempo y decidir si es conveniente incrementar o no la co- 
locación de créditos en una economía (King y Plosser 2001). Por último, Bustamante (2005) sostiene que, en la oferta de crédito, la tasa de interés afecta tanto la rentabilidad como el riesgo que asume un acreedor. Desde la perspectiva de rentabilidad, la relación es positiva con la oferta de crédito porque representa el beneficio que puede alcanzar un intermediario financiero (Domínguez y Naranjo 2009). En contraste, la tasa de interés analizada como representación del riesgo presenta una relación ambigua con la oferta de crédito, considerando que depende de la disposición de un intermediario financiero asumir riesgos para incrementar el crédito (Freimer y Gordon 1965). Mientras, Stiglitz y Weiss (1981) demostraron que la relación entre la oferta de crédito y la tasa de interés no siempre presenta una relación directa, puesto que, cuando la tasa de interés excede el punto máximo al que los acreedores están dispuestos a afrontar riesgos, la relación se invierte. A esta relación inversa los autores la denominaron como racionamiento de crédito.

\section{Riesgo bancario}

Como se mencionó anteriormente, una de las funciones bancarias es la gestión del riesgo. En consecuencia, según Düllmann y Masschelein (2007), el riesgo en el contexto bancario es la probabilidad de incurrir en pérdidas. Pyle (1997) demostró que las principales fuentes de riesgo bancario son el riesgo de mercado, el riesgo operacional y el riesgo de crédito.

El riesgo de mercado es la variación en el valor de una cartera debido a la variabilidad en los factores económicos subyacentes como las tasas de interés, los tipos de cambio y los precios internacionales de los commodities (Jahn, Memmel y Pfingsten 2013). El riesgo operacional es el riesgo de posibles efectos adversos sobre el resultado financiero del banco por efecto de fallas en procedimientos y procesos internos, omisiones -intencionales o no- en el trabajo de los empleados, así como errores cometidos en transacciones. Por último, el riesgo de crédito es la variación del valor de la cartera por el incumplimiento de la contraparte en las obligaciones adquiridas (Martin y Wilde 2002).

Skridulytė y Freitakas (2012) demostraron que el riesgo de crédito es la principal fuente de exposición que las instituciones bancarias asumen, dado que, como menciona York (2007), el otorgamiento de crédito es el servicio financiero que mayor beneficio económico representa para un banco. 
El Comité de Basilea II estableció que el riesgo de crédito en la cartera surge por el riesgo del entorno económico y el riesgo de concentración (González y Solís 2012). El primero, el riesgo del entorno económico, proviene por cambios macroeconómicos y financieros inesperados que afectan la capacidad de pago de los prestatarios (Gordy 2003). En este sentido, Vasicek (2002) argumentó que el cumplimiento de las obligaciones financieras por parte de los prestatarios está determinado por el contexto económico y financiero en el que se desempeñan sus operaciones. El segundo, el riesgo de concentración, surge por la distribución asimétrica de la cartera entre los prestatarios (concentración de créditos en relativamente pocos prestatarios) o entre sectores industriales (concentración sectorial) (Skridulyte y Freitakas 2012). La concentración sectorial de créditos expone a las instituciones bancarias, pues si los factores de riesgo que afectan a un sector económico cambian negativamente, todos los prestatarios pertenecientes a ese sector experimentarán dificultades para cubrir sus obligaciones financieras; por consiguiente, los bancos enfrentarán una gran pérdida inesperada por una situación de impago de créditos (Kuodis 2010; Adams y Leah 2006; Fiori y Lanotti 2006). Por el contrario, cuando un banco presenta una cartera de créditos diversificada, el valor esperado del incumplimiento de pago por parte de los prestatarios se va a concentrar en el valor de la pérdida promedio (figura 1). En otras palabras, a medida que una cartera presenta una mayor diversificación, la varianza del riesgo de impago de la cartera disminuye, lo que indudablemente representa un menor riesgo para el manejo bancario (Albarrán 2012). Esta relación ocurre por la propiedad de la ley de los grandes números. ${ }^{1}$

1. Cuando se tiene una base más grande, el valor esperado se concentra alrededor de la media (Pérez 2017). 
Figura 1

\section{El riesgo por concentración sectorial de créditos}

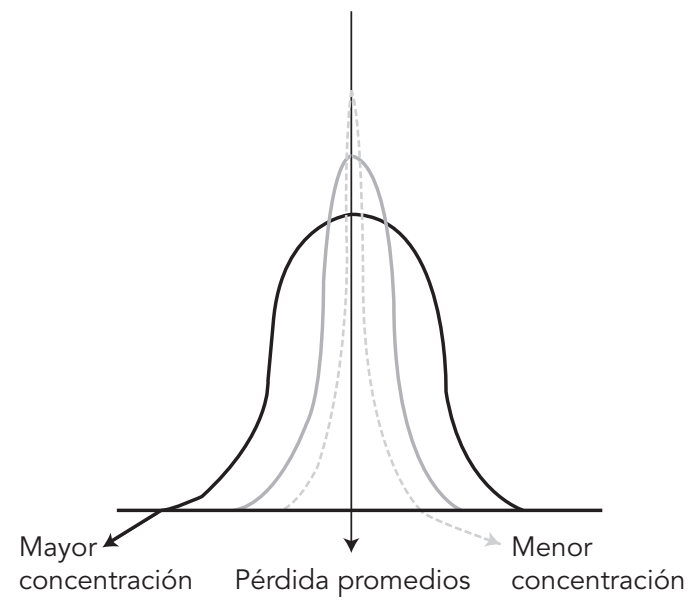

Fuente: Albarrán (2012).

Para evitar el riesgo de concentración, los bancos deben, por un lado, fijar límites a la concentración de créditos (figura 2) y, por otro lado, medir ${ }^{2}$ el riesgo de la cartera por sectores económicos de un país para mantener un manejo responsable de la institución y de los recursos depositados por los agentes, como está dispuesto en la regulación de Basilea III (BCBS 2006; Düllmann 2006; Bonti el al. 2006).

Sin embargo, la concentración sectorial de créditos surge como resultado de las características de la estructura productiva de un país, en un período de tiempo determinado. Por lo tanto, los bancos, al financiar una economía que presenta una producción centralizada en ciertos sectores económicos, implícitamente están condicionados a concentrar su cartera de créditos (Hibbeln 2010). Lütkebohmert (2009) demostró que las instituciones bancarias adecuan su cartera de créditos a partir del esquema productivo de un país,

2. Los métodos más utilizados por los bancos para medir el riesgo por concentración sectorial son: el índice Herfindahl-Hirschman, el coeficiente de Gini y los modelos de factores múltiples (Skridulytė y Freitakas 2012; Holub, Nyklíček y Pavel 2014). 
Figura 2

\section{Límites a la concentración}

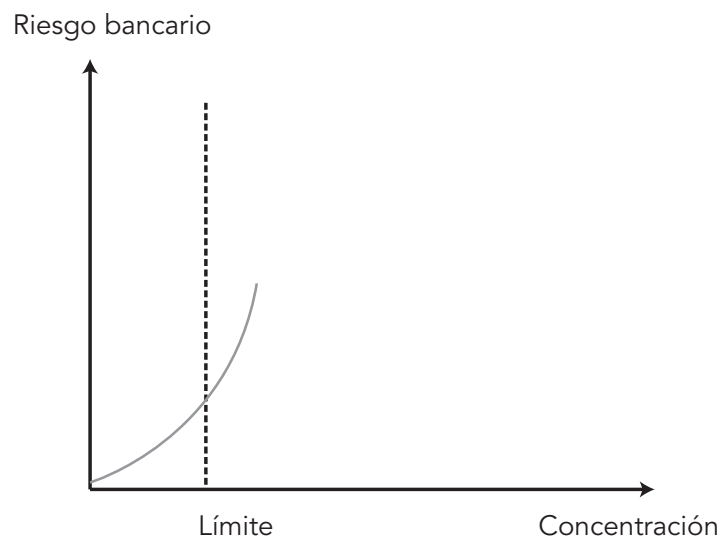

Fuente: BCBS (2006).

es decir, la composición de la cartera del banco está correlacionada con la estructura productiva de una economía, puesto que el financiamiento de las actividades más representativas es de mayor beneficio económico para los bancos.

El Comité de Basilea III definió que, en presencia de la concentración sectorial de créditos, los bancos obligatoriamente deben medir el riesgo de la cartera para evaluar que el capital y las provisiones con que cuentan son suficientes para enfrentar el riesgo generado por la concentración de cartera (González y Solís 2012). En este sentido, Holub, Nyklíček y Pavel (2014) y Skridulytė y Freitakas (2012) demostraron que las instituciones bancarias que concentraron sus créditos en el sector de manufacturas en la República Checa, para el período 2004-2014, y en el sector inmobiliario en Lituania, en el período 2004-2010, respectivamente, inevitablemente tuvieron que incrementar el capital y las provisiones para minimizar el riesgo por concentración sectorial de créditos. En consecuencia, el aumento de las provisiones de un banco repercute en menores recursos para ampliar la colocación de créditos, porque las instituciones financieras, frente al deterioro de la calidad de la cartera, incrementan el ratio capital/activo y reducen la colocación de 
crédito para minimizar el riesgo generado por concentración sectorial de créditos (Antoniades 2018; Echeverry y Salazar 1999).

Existen investigaciones que demuestran y sustentan lo mencionado anteriormente. Rodríguez y Gómez (2002) estudiaron los efectos de la focalización de los créditos otorgados por instituciones bancarias españolas en el sector inmobiliario de ese país, para el período 2009-2010. El autor demostró que la concentración crediticia por parte de los bancos expuso a las carteras de crédito a un mayor riesgo sistémico, puesto que la actividad inmobiliaria era determinante en el crecimiento económico de España, para ese período. Debido a la recesión económica que el país enfrentó, se produjo un incremento en la morosidad en el pago de los créditos; como consecuencia, las instituciones bancarias incrementaron sus provisiones y sufrieron pérdidas fuertes inesperadas, afectando de esta manera el otorgamiento de nuevos créditos. Düllmann y Masschelein (2007) desarrollaron un análisis para encontrar las causas del decrecimiento del volumen de crédito del banco alemán Deutsche Bank en el año 2004. De acuerdo con los resultados, la excesiva concentración de los créditos en el sector automovilístico, industria de mayor relevancia en la economía alemana para ese año, implicó un incremento del capital en provisiones, como medida para reducir el riesgo por concentración, provocando menores recursos para la colocación de créditos. Arroyo y Cárcamo (2009) realizaron un estudio acerca de la crisis hipotecaria $s u b$ prime en los Estados Unidos durante los años 2007-2008. Los autores demostraron que los bancos norteamericanos, desde finales de la década de los noventa, concentraron sus carteras en el sector inmobiliario, puesto que era uno de los sectores más rentables, importantes y dinamizadores de la economía estadounidense. Entre los principales hallazgos encontraron que, previo a la crisis financiera y bancaria, el incumplimiento en el pago de hipotecas generó un mayor aprovisionamiento por parte de los bancos en cada crédito otorgado, reduciendo los recursos disponibles para financiar la economía.

Por último, Portocarrero y Tarazona (2004) estudiaron la reducción del crédito otorgado por las cajas rurales de ahorro y crédito (CRAC) en Perú entre los años 1993 y 1996. Los autores encontraron que más del 75\% de la cartera de créditos estaba concentrada en el sector agrícola debido al giro de negocio de estas instituciones, y que, como consecuencia de los shocks externos (inundaciones) que afectaron a ese sector en aquellos años, estas 
instituciones tuvieron que incrementar las provisiones, como medida para reducir las pérdidas por el impago generalizado de los créditos por parte de los prestatarios, lo que generó una fuerte contracción del crédito.

\section{Contexto}

\section{Volumen de crédito en América Latina}

En América Latina, la colocación de crédito se ha caracterizado por su volatilidad y por responder fácilmente a shocks externos económicos (CEPAL 2004). En la región, las expansiones del crédito ocurren como consecuencia de fuertes incrementos de los depósitos a la vista, pues es una variable fundamental en el financiamiento del crédito bancario (Harbo y Sulla 2013). En América Latina, el crédito ha crecido a una tasa promedio anual de $5,45 \%$ entre 2004-2016 y muestra comportamientos distintos entre subperídos. En un inicio, en los años previos a la crisis financiera mundial (20042006), el crédito presentó una tasa de crecimiento anual promedio superior al $6 \%$; posteriormente, durante la época de crisis (2007-2008), la colocación de crédito presenció una fuerte contracción hasta llegar a niveles de crecimiento menores al 1\%. Para el año 2009, el crédito logró una recuperación, y hasta el 2011 creció, en promedio, 7,37\%. Finalmente, desde 2012 hasta 2016, el volumen de crédito en la región redujo su crecimiento al 3,7\%.

En América Latina, la participación de la colocación de crédito en la producción se incrementó como resultado de las altas tasas de crecimiento que el crédito mantuvo a partir del año 2002 en la región (Hansen y Sulla 2013). Los datos (figura 3) muestran que el volumen de crédito como porcentaje del PIB (participación en la producción) en la región pasó de $47 \%$ en 2004, a $62 \%$ en 2016.

Como se evidencia en la figura 4, entre 2004 y 2016, las economías que presentaron un decrecimiento de la participación del crédito en su producción fueron: Argentina, Uruguay, Nicaragua y Panamá. Por el contrario, en las economías de Costa Rica, Brasil y Chile, el crédito aumentó considerablemente su participación en el PIB, al incrementarse 35, 38 y $44 \%$, respectivamente, entre 2004 y 2016. 
Figura 3

Crecimiento del crédito en América Latina (2004-2016)

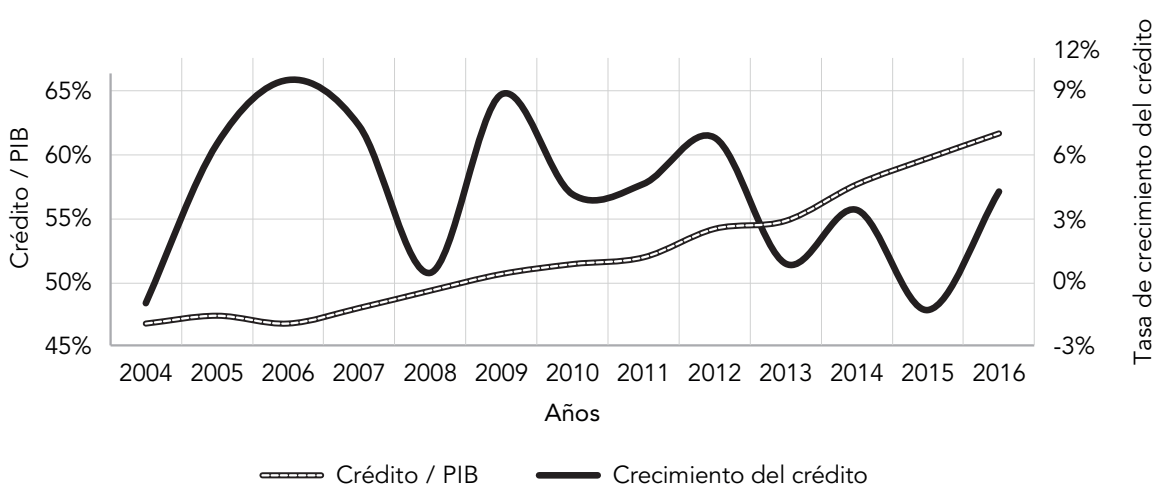

Fuente: Banco Mundial (2017).

Figura 4

\section{La colocación de crédito como porcentaje del PIB en América Latina}

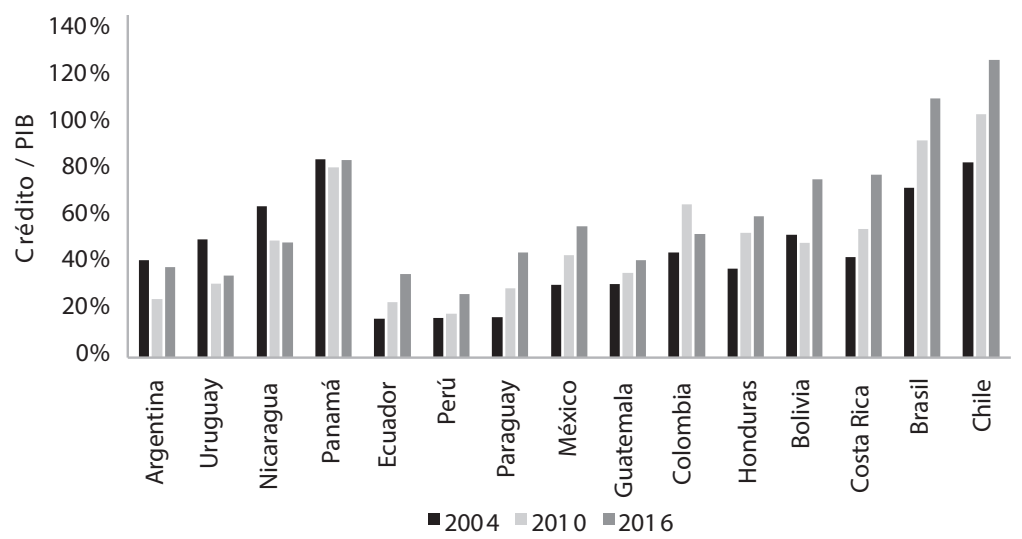

Fuente: Banco Mundial (2017).

Referente a la estructura productiva latinoamericana, Prebisch (1981) a principios de los años ochenta determinó que el principal desafío de la región será el fomento de una mayor diversificación del aparato productivo, con el objetivo de reducir la brecha existente, en productividad, con los 
Figura 5

\section{Estructura productiva de América Latina (2004-2016)}

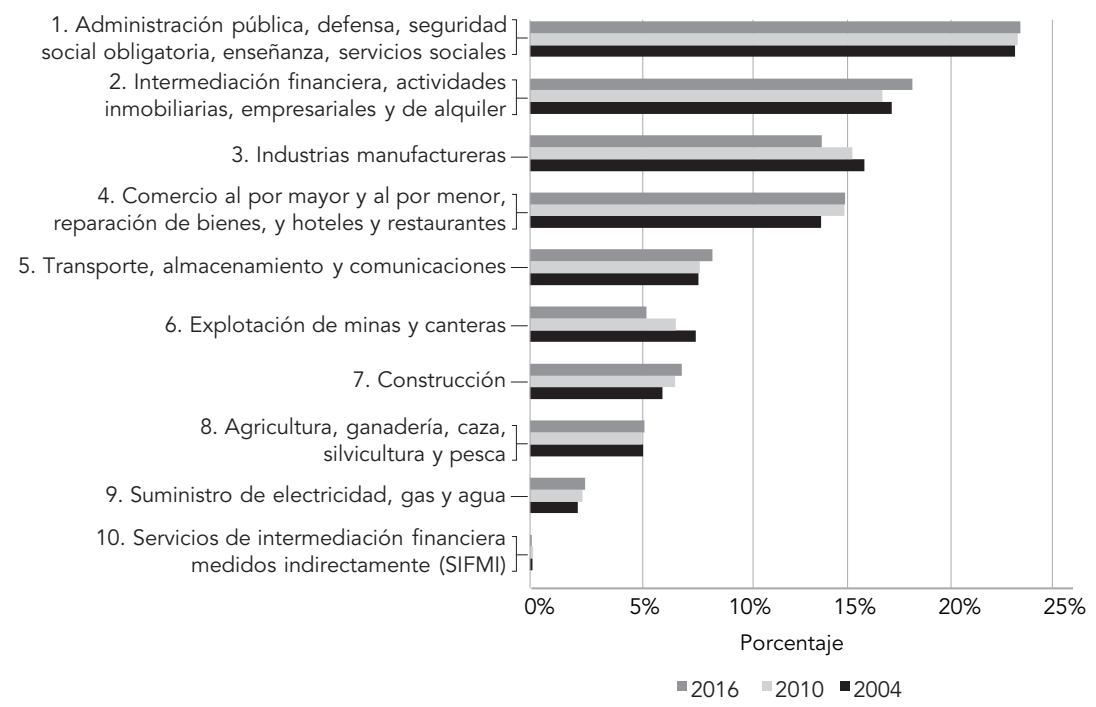

Nota. Los valores corresponden a 2016.

Fuente: CEPAL (2017).

países desarrollados. En años posteriores, según Mohanty, Schnabel y García (2006), la región no ha logrado una diversificación productiva, pues aún mantiene concentrada la producción en sectores que presentan un escaso valor agregado. De acuerdo con Imbs y Wacziarg (2002), los países más pobres se han caracterizado por mantener niveles altos de concentración sectorial en su producción. En la figura 5 se demuestra la evolución de la estructura productiva de América Latina en el período 2004-2016.

Según los datos de la CEPAL (2017), entre 2004 y 2016, el valor agregado de la producción latinoamericana se aglomera en cuatro sectores económicos: 1. administración pública, defensa, enseñanza y salud $(23,35 \%)$; 2. intermediación financiera, actividades inmobiliarias y empresariales $(18,21 \%)$; 3. industrias manufactureras $(13,89 \%)$; $y$, 4. comercio al por mayor y menor $(15,02 \%)$. En conjunto, estos sectores económicos representaron, en promedio, más del $70 \%$ del valor agregado de la región en todos los años del período analizado. 
Al analizar la estructura productiva privada de la región, es decir, sin tomar en cuenta el primer sector (administración pública, defensa, enseñanza y salud), la región concentra cerca del 50\% del valor agregado de la producción privada en los tres sectores económicos restantes, entre 2004 y 2016. Otro indicador para medir el grado de concentración productiva es el índice Herfindahl-Hirschman ( $\mathrm{IHH})$, mismo que toma valores entre 0 y 1.800 puntos en adelante, y su análisis es el siguiente:

Tabla 1

Índice Herfindahl Hirschman

\begin{tabular}{|l|l|}
\hline \multicolumn{2}{|c|}{$\begin{array}{c}\text { Criterio índice Herfindahl Hirschman } \\
\text { (medido en puntos) }\end{array}$} \\
\hline Intervalo & Nivel de concentración \\
\hline $0-1.000$ & Economía diversificada \\
\hline $1.000-1.800$ & Economía moderadamente concentrada \\
\hline 1.800 en adelante & Economía altamente concentrada \\
\hline
\end{tabular}

Fuente: Departamento de Justicia de los Estados Unidos (2015).

Elaboración propia.

De acuerdo con los resultados (tabla 2), América Latina presentó un IHH en el período 2004-2016, en promedio, de 1.555,4 puntos. De igual modo, se encontró que América del Sur tiene un menor nivel de concentración en su producción (con un índice de 1.506,5 puntos) en comparación a América Central (con 1.604,6 puntos). Sin embargo, de acuerdo con el criterio del IHH, la región latinoamericana manifiesta una concentración moderada en su producción.

Tabla 2

Promedio del IHH de cada región (2009-2016) expresada en puntos

\begin{tabular}{|l|l|}
\hline América Latina & $1.555,44$ \\
\hline América del Sur & $1.506,26$ \\
\hline América Central & $1.604,61$ \\
\hline
\end{tabular}

Fuente: CEPAL (2017). 
Los países que alcanzaron el máximo IHH en el año 2016 fueron Brasil y Honduras, con 1.826,47 y 1.837,83 puntos, respectivamente; por el contrario, las economías que presentaron el menor índice fueron Ecuador y Guatemala con $1.263,48$ y $1.451,52$ puntos, respectivamente. La evolución del IHH en los países de la región, entre 2004 y 2016, ha sido heterogénea, siendo Honduras y Costa Rica las economías en donde menos desconcentración productiva sucedió, pues el IHH se incrementó en $164(9,8 \%)$ y 106 puntos (6,4\%), respectivamente; mientras que en países como Bolivia y Ecuador, la concentración productiva se redujo y eso se refleja en decrementos de $155(9,5 \%)$ y 124 (9\%) puntos, respectivamente, en el IHH en los 12 años de estudio. Adicionalmente, economías como Brasil y Paraguay se mantuvieron sin cambios importantes en su estructura productiva, al presentar una variación de $0,21 \% \mathrm{y}$ $-0,81 \%$, respectivamente, entre 2004 y 2016.

Finalmente, al analizar el comportamiento de la concentración productiva y la colocación de crédito en la región entre 2004 y 2016 (figura 6), se halló que en dos períodos las variables presentaron una relación coincidente con el planteamiento teórico. Para el primer período, es decir, entre 2006 y 2007, la región presentó un incremento en su concentración productiva en 2,89 puntos, lo que estuvo acompañado de una contracción del crédito en 2,33 puntos. El segundo período de coincidencia teórica fue entre 2009 y 2010, cuando el crédito decreció 5,09 puntos, mientras que la concentración en la producción latinoamericana se incrementó 1,86 puntos.

\section{Metodología}

El estudio de los determinantes de la oferta de crédito ha sido, históricamente, fundamental para entender, explicar y predecir el comportamiento del crédito en momentos de auge, crecimiento, recesión y crisis que enfrentan las economías en el mundo (Barajas y Steiner 2002). En este sentido, la mayoría de investigaciones empíricas coinciden en la inclusión de variables como la actividad económica, el nivel de depósitos, la tasa de interés real, y, para ciertas regiones, los precios de commodities energéticos, ${ }^{3}$ para explicar la variabilidad de la colocación de crédito en determinadas regiones y países.

3. Nafta, etanol, petróleo crudo, fuel oil, gas. 
Figura 6

\section{La concentración productiva y la colocación de crédito en América Latina}

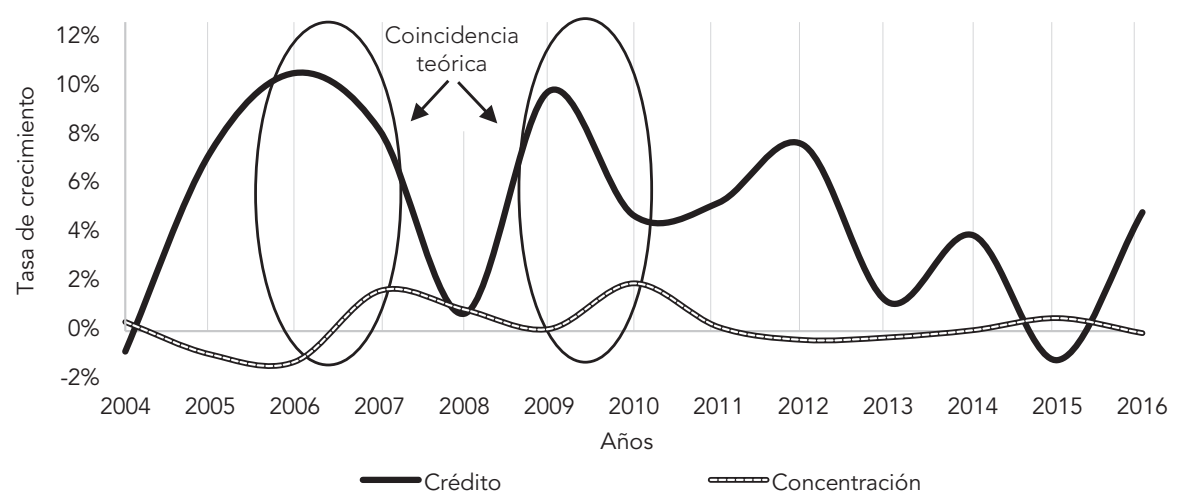

Fuente: CEPAL (2017).

Para el caso de la actividad económica, autores como Guo y Stepanyan (2011), Favara e Imbs (2015), y Gilchrist y Zakrajšek (2012) han concluido que el PIB real es fundamental para explicar el crédito, pues consideran que es el reflejo de la salud de la economía y, bajo un escenario de altas tasas de crecimiento de esta variable, los acreedores presentan una mayor predisposición para financiar el incremento del consumo e inversión por parte de los agentes económicos.

Con respecto a los depósitos a la vista, Mohanty, Schnabel y García (2006) y Anabtawi y Smith (1994) incluyen esta variable en sus modelos de crédito y demuestran que los depósitos son la principal fuente de fondeo que mantienen las instituciones bancarias para ampliar la colocación de crédito y afirman la existencia de una relación positiva entre ambas variables. Sin embargo, Guo y Stepanyan (2011) hallaron que, para ciertas economías emergentes de América Latina, en períodos poscrisis los depósitos a la vista pierden relevancia como fuente de fondeo bancario y el endeudamiento externo se vuelve significativo para la oferta crediticia.

En relación a la tasa de interés real, Rodríguez (1996) realizó una investigación acerca del desenvolvimiento de la banca en México en los años 
noventa y demostró que, conforme la tasa de interés real se incrementaba, también lo hacía la colocación de créditos. De igual manera, Soss (1993) y Steindl (2000) demostraron que los acreedores presentaban mayores beneficios al otorgar créditos cuando la tasa de interés real era elevada. Sin embargo, Cheng y Xu (2004) encontraron que la tasa de interés real oficial en China para el año 1996 fue tan alta que redujo el crédito para el sector rural, pues para los acreedores el riesgo era superior a los beneficios que podían alcanzar. $^{4}$

Respecto a los precios de los commodities energéticos, en un estudio sobre los efectos del precio real del petróleo en las principales variables macroeconómicas de países exportadores e importadores de crudo, Steindl (2000) encontró que, ante incrementos de los precios del petróleo, los países exportadores de crudo presentan mayores niveles de liquidez en sus economías, lo que dinamiza la colocación de créditos por parte de los bancos. Jaramillo, Lehmann y Moreno (2009) demostraron que en determinados países latinoamericanos los precios de los commodities son una variable significativa para el incremento del crédito.

Con base en la discusión anterior, el modelo de datos de panel, de periodicidad anual, planteado en esta investigación, utiliza las variables descritas en la ecuación 1 para comprobar que la concentración productiva presenta una relación negativa con la colocación de crédito en quince países de América Latina, siendo nueve sudamericanos ${ }^{5}$, excluyendo a Venezuela, ${ }^{6}$ y seis centroamericanos, ${ }^{7}$ para el período 2004-2016.

$$
\begin{aligned}
& a C r \text { édito } i j=\beta 0+\beta 1\left(\text { aPIBreal }_{i j}\right)+\beta 2\left(\text { aDepósitos }_{i j}\right)+\beta 3\left(\text { dif Interés real }_{i j}\right) \\
& +\beta 4(\text { tc Precio Petróleo } i j)-\beta 5\left(\text { L. dif Índice } H H_{i j}\right)+u_{i j}
\end{aligned}
$$

La aceleración del volumen de crédito (aCrédito) es la variable dependiente y es explicada por el producto interno bruto en términos constantes (a PIB real) y los depósitos a la vista reales (aDepósitos), ambos expresados

4. Esta relación coincide con la teoría del racionamiento del crédito propuesta por Stiglitz y Weiss (1981).

5. Argentina, Brasil, Bolivia, Chile, Colombia, Ecuador, Paraguay, Perú y Uruguay.

6. País que presenta observaciones atípicas y falta de disponibilidad de datos.

7. Costa Rica, Honduras, Guatemala, Nicaragua, México y Panamá. 
en aceleración, la primera diferencia de la tasa de interés real (dif Interés Real), la tasa de crecimiento del precio del barril de petróleo real (tc Precio Petróleo) y la primera diferencia del índice Herfindahl-Hirschman con un rezago (L. dif Índice $H H$ ). El término $i$ indica el individuo (país) y $j$ el tiempo. El índice Herfindahl Hirschman $(I H H)$ es una medida generalmente aceptada para establecer el nivel de concentración en un mercado (Ayala 2015) y resulta de la sumatoria al cuadrado de las cuotas de mercado $(\mathrm{Si})$ de $n$ industrias, empresas o ramas económicas (Hirschman 1964). Para la elaboración de este indicador se empleó las estadísticas publicadas por CEPAL de las 10 principales actividades productivas ${ }^{8}$ que componen el valor agregado (ecuación 2).

\section{índice Herfindahl Hirschman $=\sum_{i=1}^{n} S i^{2}$}

Como se mencionó en la tabla 1, el índice se expresa en puntos ${ }^{9}$ en una escala de 0 en adelante (Departamento de Justicia de los Estados Unidos 2015). ${ }^{10}$

El volumen de crédito y la tasa de interés real fueron tomados de la base de datos del Banco Mundial; el PIB real y los depósitos a la vista de la CEPAL y el precio de petróleo del Fondo Monetario Internacional. Las variables están expresadas en términos reales con año base 2010, a excepción del precio del petróleo, que tiene como año base 2005 .

El modelo estimado por datos de panel, con errores estándar robustos, para un número de 180 observaciones presentó un nivel de ajuste general (overall) de

8. Las diez ramas de la economía tomadas en cuenta son: 1. Agricultura, ganadería, caza, silvicultura y pesca. 2. Explotación de minas y canteras. 3. Industrias manufactureras. 4. Suministro de electricidad, gas y agua. 5. Construcción. 6. Comercio al por mayor y al por menor, reparación de bienes, y hoteles y restaurantes. 7. Transporte, almacenamiento y comunicaciones. 8. Intermediación financiera, actividades inmobiliarias, empresariales y de alquiler. 9. Administración pública, defensa, seguridad social obligatoria, enseñanza, servicios sociales y de salud, y otros servicios comunitarios, sociales y personales. 10. Servicios de intermediación financiera medidos indirectamente (SIFMI 2008).

9. Mientras más alta sea la puntuación del índice, más concentración productiva existirá en una economía.

10. La mayoría de las investigaciones empíricas se fundamentan en el criterio del Departamento de Justicia de los Estados Unidos. 
$18,83 \%,{ }^{11}$ es decir, las variables independientes explican un $18,83 \%$ la variación del volumen de crédito. Mientras que el ajuste entre cada uno de los países y las variables (within) fue de $18,83 \%$ y el ajuste del modelo entre las variables (between) de 24,89\%.

Todas las variables incluidas en el modelo son significativas al menos al 95\% de confianza, con excepción del índice Herfindahl Hirschman que se acepta a un $90 \%$ de confianza. Por otra parte, los signos de los coeficientes de las variables presentan una relación esperada con el volumen de crédito (tabla 3).

Tabla 3

Resultados del modelo econométrico

\begin{tabular}{|c|c|c|c|}
\hline & $\mathrm{R}^{2}$ within: & 0,1883 & N. observaciones: 180 \\
\hline & $\mathrm{R}^{2}$ between: & 0,2489 & \\
\hline & $\mathrm{R}^{2}$ overral: & 0,1883 & \\
\hline Variables & Coeficiente & $\begin{array}{l}\text { Error estándar } \\
\text { robusto }\end{array}$ & Z \\
\hline PIB real & 0,0034948 & 0,001612 & $2,17^{* * *}$ \\
\hline Depósitos & 0,126008 & 0,062062 & $2,03^{* * *}$ \\
\hline dif. interés real & 0,0091414 & 0,0028671 & $3,19^{* * *}$ \\
\hline TC precio petróleo & 0,0767179 & 0,0398675 & $1,92^{* *}$ \\
\hline L. dif. índice HH & $-0,000795$ & 0,0004619 & $-1,72^{*}$ \\
\hline Constante & 0,0022849 & 0,0022849 & 0,36 \\
\hline
\end{tabular}

Nota. Niveles de confianza: $\left(^{*}\right) 90 \%,\left({ }^{* *}\right) 95 \%$, y $\left(^{* * *}\right) 99 \%$.

Elaboración propia.

Con el objetivo de contar con mejores estimadores lineales insesgados (MELI), se procedió a realizar pruebas de robustez del modelo. En primer

11. En estudios semejantes realizados (Guo y Stepanyan 2011) se obtuvieron ajustes globales del 23 y $30 \%$, respectivamente. El ajuste en esta investigación es menor porque se utilizaron menos países. 
lugar, se realizó el test de Hausman para determinar el método de estimación más apropiado. Al obtener un valor de 0,9951 se rechazó la hipótesis nula y el modelo se estimó con efectos aleatorios ${ }^{12}$ (tabla 4).

Tabla 4

\section{Test de Hausman}

\begin{tabular}{|l|}
\hline Test de Ho: diferencia en los coeficientes no es sistemática. \\
\hline Chi2 $=0,41$ \\
\hline P valor $=0,9951$ \\
\hline
\end{tabular}

Elaboración propia.

Consecutivamente, se comprobó que las variables presentan un comportamiento estacionario. Además, se realizó el test de raíz unitaria conjunta Levin-Lin-Chu que permitió confirmar que todas las variables incluidas en el modelo son estacionarias (tabla 5).

Tabla 5

Prueba conjunta de raíz unitaria

\begin{tabular}{|l|c|l|c|}
\hline Variable & $\begin{array}{c}\text { Valor } \mathbf{p} \text { a } \\
\text { nivel }\end{array}$ & \multicolumn{1}{|c|}{ Tratamiento } & Valor $\mathbf{p}$ \\
\hline Crédito & 0,25896 & Aceleración & 0,00000 \\
\hline PIB real & 0,12386 & Aceleración & 0,00000 \\
\hline Depósitos & 0,11589 & Aceleración & 0,00000 \\
\hline Interés real & 0,36587 & Primera diferencia & 0,00000 \\
\hline Precio petróleo & 0,75369 & Tasa de crecimiento & 0,00045 \\
\hline Índice HH & 0,36985 & Primera diferencia & 0,00770 \\
\hline
\end{tabular}

Elaboración propia.

12. El modelo de efectos aleatorios considera que los efectos individuales no son independientes entre sí, sino que están distribuidos aleatoriamente alrededor de un valor dado (Baronio, Vianco y Rabanal 2012). 
Posteriormente, las pruebas realizadas comprueban que el modelo presentó homocedasticidad en los residuos y no autocorrelación serial de los errores (tabla 6). Finalmente, para comprobar la no presencia de multicolinealidad entre las variables explicativas, se realizó la matriz de correlación y se comprobó que no existe correlación mayor a 0,5 , de manera que se descartó la presencia de multicolinealidad en el modelo (tabla 7).

Tabla 6

Pruebas de robustez

\begin{tabular}{|c|c|c|}
\hline Test & Ho & Resultado \\
\hline $\begin{array}{c}\text { Breusch } \\
\text { Pagan }\end{array}$ & Modelo presenta homocedasticidad & $\begin{array}{c}\text { (Valor Prueba }=9)<(\text { Valor } \\
\text { Crítico }=12,59)\end{array}$ \\
\hline Wooldridge & Modelo no presenta autocorrelación & Valor $p=0,068$ \\
\hline
\end{tabular}

Elaboración propia.

Tabla 7

Matriz de correlación

\begin{tabular}{|l|r|r|r|r|r|l|}
\hline & Crédito & PIB real & $\begin{array}{c}\text { Interés } \\
\text { real }\end{array}$ & Depósitos & $\begin{array}{c}\text { Precio } \\
\text { petróleo }\end{array}$ & IHH \\
\hline Crédito & 1 & & & & & \\
\hline PIB real & 0,166 & 1 & & & & \\
\hline Interés real & 0,2643 & $-0,111$ & 1 & & & \\
\hline Depósitos & 0,2039 & 0,267 & $-0,0588$ & 1 & & \\
\hline $\begin{array}{l}\text { Precio } \\
\text { petróleo }\end{array}$ & 0,167 & 0,3031 & $-0,195$ & 0,1091 & 1 & \\
\hline $\mathrm{IHH}$ & $-0,1538$ & $-0,0073$ & 0,0215 & 0,0267 & $-0,1181$ & 1 \\
\hline
\end{tabular}

Elaboración propia. 


\section{Resultados}

Los resultados obtenidos confirman que la concentración productiva presenta una relación negativa con el volumen de crédito en las economías latinoamericanas entre 2004 y 2016. Es decir, cuanto más concentrada sea una estructura productiva, menor es el crédito que se otorga.

De acuerdo con los coeficientes hallados, el aumento en un punto del índice de concentración productiva en el período anterior desacelera el crédito en el período actual en -0,000795 puntos. Este resultado es coincidente con el encontrado por Alvarado (1984), quien demostró que en Perú, entre 1983 y 1984, los bancos especializados en el sector agrario reducían la oferta de créditos para el financiamiento de proyectos que dependían de uno o pocos cultivos, debido al riesgo que representaba para los acreedores la concentración en la producción.

Respecto a los estimadores fundamentales del crédito, se obtuvieron los resultados consistentes con la teoría. En primer lugar, se encontró que la aceleración del PIB real en $1 \%$ causa una aceleración del volumen de crédito en $0,003498 \%$. Este resultado es similar con la investigación realizada por Guo y Stepanyan (2012) al comprobar que, en economías emergentes, altos niveles de crecimiento del PIB son determinantes en la colocación de crédito, puesto que las instituciones financieras satisfacen el aumento de consumo e inversión por parte de los agentes económicos.

De igual manera, los resultados del efecto de la tasa de interés en la colocación de crédito cumplen con la teoría. Puntualmente, el incremento de la tasa de interés real en $1 \%$ acelera el crédito en 0,0091414\%. Esto es similar con los resultados hallados para Filipinas por Tan (2012), donde la oferta de crédito aumentó conforme lo hizo la tasa de interés real al representar mayores rendimientos para los acreedores.

Con respecto a los depósitos a la vista, los resultados demuestran el cumplimiento de la relación teórica con la oferta de crédito. Precisamente, la aceleración de los depósitos en un $1 \%$ genera una aceleración del crédito en $0,126008 \%$. En este sentido, Murcia y Piñeros (2006) demostraron que, conforme se incrementaban los depósitos a la vista en Colombia en el período 1998-1999, los bancos contaban con mayor liquidez para incrementar la oferta de créditos. 
Finalmente, los resultados confirman la importancia del precio del petróleo en la oferta de crédito en la región, puesto que los quince países seleccionados en esta investigación se clasifican como exportadores de combustibles y lubricantes (CEPAL 2004). Concretamente, si la tasa de crecimiento del precio del petróleo aumenta $1 \%$, el crédito se acelera en 0,0767179\%. Esto es semejante con los resultados encontrados para países exportadores de petróleo por Lanteri (2014) al demostrar que, cuando la cotización del petróleo sube, los bancos incrementan los volúmenes de crédito como consecuencia del aumento de la demanda de crédito causada por mejores niveles de actividad económica y empleo.

\section{Conclusiones}

\section{$y$ recomendaciones}

Las estimaciones permiten demostrar que, entre 2004 y 2016, en quince países latinoamericanos (nueve sudamericanos, cinco centroamericanos y uno norteamericano), la colocación de crédito se desacelera como respuesta a incrementos en la concentración de la estructura productiva, medida a través del índice Herfindahl Hirschman (IHH). Esta relación puede explicarse porque las instituciones bancarias al desempeñarse en economías con baja diversificación productiva, están condicionadas a concentrar sus carteras de créditos en aquellos sectores económicos representativos, resultados similares obtenidos por Hibbeln (2010). Varios autores (Albarrán 2012; Antoniades 2018; Echeverry y Salazar 1999) sostienen que, como consecuencia, las instituciones bancarias asumen un mayor riesgo por concentración sectorial de créditos; es decir, la varianza del riesgo de impago de la cartera de crédito aumenta por ausencia de diversificación del portafolio. Para minimizar este riesgo, los bancos incrementan el aprovisionamiento por cada desembolso realizado, lo que genera menores recursos para incrementar los créditos.

Por otra parte, de acuerdo con el índice de concentración calculado se comprueba que el $70 \%$ del valor agregado de la producción latinoamericana se agrupa en cuatro sectores económicos. Esta característica de la región se mantuvo durante el período de estudio. 
Adicionalmente, de acuerdo con las estimaciones en América Latina durante el período 2004-2016, la aceleración de la colocación de crédito presenta una relación directa con la actividad económica, puesto que, ante un mejor desempeño económico, los acreedores presentan una mayor predisposición para financiar el incremento del consumo e inversión por parte de los agentes económicos. El nivel de depósitos también presenta una relación directa, puesto que son la principal fuente de fondeo que tienen los bancos para otorgar créditos. La tasa de interés presenta una relación positiva, dado que representa la rentabilidad que puede alcanzar el acreedor; sin embargo, es importante mencionar que esta relación puede invertirse, pues la tasa de interés también materializa el riesgo a asumir por el prestamista, dando lugar a un racionamiento del crédito. Por último, el precio del petróleo, variable proxy de los commodities energéticos, registró un vínculo directo con la aceleración de la oferta de crédito, pues ante incrementos en su cotización, aumentan los niveles de liquidez en las economías, lo que dinamiza la oferta de créditos por parte de los bancos. En este sentido, los resultados obtenidos son esperados, puesto que son las variables fundamentales de la colocación de crédito.

La baja diversificación productiva, que está asociada a la concentración de cartera, es un problema para el manejo y administración bancario. En efecto, para revertir el impacto negativo que presenta la concentración productiva sobre la colocación de crédito, relación demostrada en esta investigación, se recomienda como política pública la generación de mecanismos para incentivar a las instituciones bancarias el financiamiento de los sectores económicos de mayor riesgo debido a su minoritaria participación en la producción, como por ejemplo, la creación de una normativa que permita a los gobiernos de la región cubrir una parte de las tasas de interés que los bancos cobran a los prestatarios pertenecientes a esos sectores. De igual manera, para países donde existen tasas máximas de interés como Ecuador, Colombia y Chile, se recomienda que los organismos de control financiero incrementen los techos de las tasas de interés a los que están sujetos los créditos, como un mecanismo de estímulo hacia los bancos para incrementar el financiamiento de las actividades de los sectores económicos de menor contribución en la producción. 


\section{Referencias}

Adams, Tony, y Kelly Leah. 2006. "Measuring Credit Portfolio Risk". Economics \& Sociology 5 (1): 51-61. 〈http://bit.ly/2Wdp3Cq〉.

Akerlof, George. 1970. "The Market for 'Lemons': Quality Uncertainty and the Market Mechanism”. The Quarterly Journal of Economics 84 (3): 488-500. Doi: 10.2307/1879431.

---, y Robert Shiller. 2009. Animal spirits: cómo influye la psicología humana en la economía. Madrid: Gestión 2000.

Albarrán, Irene. 2012. "La actividad aseguradora: importancia, revisión e integración de conceptos fundamentales". Documentos de trabajo, Facultad de Ciencias Económicas y Empresariales, Universidad Complutense de Madrid. 〈http://bit.ly/2RDel7L〉.

Alexander, William, y Francesco Caramazza. 1994. "Money $t$ Versus Credit: The Role of Banks in the Monetary Transmission Process". En Frameworks for Monetary Stability: Policy Issues and Country Experiencies, 397-422. Washington D. C.: IFM.

Anabtawi, Iman, y Gary Smith. 1994. "Macroeconomic Modeling of Money, Credit, and Banking”. Eastern Economic Journal 20 (3): 275-290. 〈http://bit.ly/31lenCD〉.

Antoniades, Adonis. 2018. "Uneven Recovery: Mortgage Credit and the Concentration Channel”. Journal of Finance 64 (2): 861-922. 〈https://papers.ssrn.com/sol3/papers.cfm?abstract_id=3248072>.

Arroyo, María del Pilar, y Ester Cárcamo. 2009. "La crisis hipotecaria de Estados Unidos y sus repercusiones en México". Economía y Sociedad 14 (24): 93-104. 〈https://www. redalyc.org/pdf/510/51015096006.pdf).

Audretsch, David, Werner Bönte y Mahagaonkar Prashanth. 2009. "Financial Signalling by Innovative Nascent Entrepreneurs”. Discussion Paper No. 7165. Londres: Centre for Economic Policy Research. 〈http://bit.ly/2RBoBNu〉.

Ayala, Eduardo. 2015. "El Índice Herfindahl-Hirschman (HHI)". Gerencia de Mercadeo: Ciencias Económicas y Administrativas. Consulta: abril de 2019. «http://3w3search.com/ Edu/Merc/Es/GMerc064.htm〉.

Barajas, Adolfo, y Roberto Steiner. 2002. "Why Don't They Lend? Credit Stagnation in Latin America”. IMF Staff Papers, 49: 156-184. Consulta: febrero de 2019. 〈http://bit. ly/2oilawX>.

Baronio, Alfredo, Ana Vianco y Cristian Rabanal. 2012. "Una introducción a la econometría espacial: dependencia y heterogeneidad". Cátedra de Econometría. Consulta: mayo de 2019. 〈http://bit.ly/2MkwpAC〉.

Basel Committee on Banking Supervision (BCBS). 2004. Bank Failures in Mature Economies. Documento de trabajo No. 13. Consulta: mayo de 2019. 〈http://www.bis.org/publ/ bcbs_wp13.pdf>.

Bencivenga, Valerie, y Bruce Smith. 1991. "Financial Intermediation and Endogenous Growth". Review of Economic Studies 58 (2): 195-209. Doi: 10.2307/2297964. 
Bernanke, Ben, y Mark Gertler. 1986. “Agency Costs, Net Worth, and Business Fluctuations”. American Economic Review 79 (1): 14-31. 〈https://www.jstor.org/stable/1804770〉.

Blinder, Alan. 1987. "Credit Rationing and Effective Supply Failures”. The Economic Journal 97 (386): 327-352. Doi: 10.2307/2232882.

Bonti, Gabriel, Michael Kalkbrener, Christopher Lotz y Gerhard Stahl. 2006. "Credit Risk Concentrations under Stress". Journal of Credit Risk 2 (3): 115-136. Doi: 10.21314/ JCR.2006.042.

Bustamante, Rafael. 2005. "El problema del racionamiento de crédito en el sistema bancario peruano: como factor explicativo fundamental del costo de crédito". Tesis de pregrado, Universidad Nacional Mayor de San Marcos, Lima. Consulta: mayo de 2009. 〈http://bit. ly/31qBfAZ>.

Catarineu, Eva, y Daniel Pérez. 2008. "La titulización de activos por parte de las entidades de crédito: el modelo español en el contexto internacional y su tratamiento desde el punto de vista de la regulación prudencial”. Estabilidad financiera 14 (2): 89-121. 〈https://dialnet. unirioja.es/servlet/articulo?codigo $=2656680$ ).

Cheng, Enjiang, y Zhong Xu. 2004. "Rates of Interest, Credit Supply and China's Rural Development". Savings and Development 28 (2): 131-156. 〈https://bit.ly/33Crxg4〉.

Comisión Económica para América Latina y el Caribe (CEPAL). 2004. Implicaciones de la política macroeconómica, los choques externos y los sistemas de protección social en la pobreza, la desigualdad y la vulnerabilidad. Santiago: CEPAL.

Domínguez, Ruth, e Irina Naranjo. 2009. "Determinantes de las tasas de interés en el Ecuador con dolarización oficial. Período 2003-2007”. Tesis de pregrado, Escuela Politécnica Nacional del Ecuador. Consulta: mayo de 2019. «https://bibdigital.epn.edu.ec/handle/15000/153>.

Düllmann, Klaus. 2006. Measuring Business Sector Concentration by an Infection Model. Berlín: Deutsche Bundesbank. Consulta: abril de 2019. 〈https://bit.ly/2oMnNde〉.

---, y Nancy Masschelein. 2007. “A Tractable Model to Measure Sector Concentration Risk in Credit Portfolios”. Journal of Financial Services Research 32 (1-2): 55-79. Doi: 10.1007/ s10693-007-0014-3.

Echeverry, Juan Carlos, y Natalia Salazar. 1999. “¿Hay un estancamiento en la oferta de crédito?". Archivo de Macroeconomía. Documento 4. Consulta: enero de 2019. 〈http://bit. ly/2Ng1hSa〉. 〈https://bit.ly/2S0rZAL〉.

Favara, Giovanni, y Jean Imbs. 2015. "Credit Supply and the Price of Housing”. The American Economic Review 105 (3): 958-992. 〈https://www.jstor.org/stable/43495408〉.

Fiori, Roberta, y Simonetta Ianotti. 2006. "Estimating Macroeconomic Credit Risk and Sectoral Default Rate Correlations for the Italian Economy”. SSRN Electronic Journal, 1-55. Doi: 10.2139/ssrn.1792562.

Freimer, Marshall, y Myron Gordon. 1965. "Why Bankers Ration Credit? The Quarterly". Journal of Economics 73 (3): 390-415. Doi:10.2307/1882705. 


\section{Nicolás Barriga}

Freixas, Xavier, y Jean-Charles Rochet. 1999. Economía bancaria. Barcelona: Antonio Bosh.

Friedman, Milton. 1957. A Theory of the Consumption Function: A study by the National Bureau of the Economic Research. Nueva Jersey: Princeton University Press.

Gilchrist, Simon, y Egon Zakrajšek. 2012. "Credit Spreads and Business Cycle Fluctuations". The American Economic Review 102 (4): 1692-1720. Doi: 10.1257/aer.102.4.1692.

González, Andrés, y Ricardo Solís. 2012. "El ABC de la regulación bancaria de Basilea”. Revista Análisis Económico 27 (64): 105-139. 〈https://www.redalyc.org/pdf/413/41324545006. pdf〉.

Gordy, Michael. 2003. "A Risk Factor Model Foundation for Ratings-Based Bank Capital Rules”. Journal of Financial Intermediation 12 (5): 199-232. 〈https://bit.ly/321Doiv〉.

Greenwood, Jeremy, y Boyan Jovanovic. 1990. "Financial Development, Growth and the Distribution of Income”. Journal of Political Economy 98 (5): 1076-1107. 〈http://piketty. pse.ens.fr/files/GreenwoodJovanovicJPE1990.pdf).

Guo, Kai, y Vahram Stepanyan. 2011. "Determinants of Bank Credit in Emerging Market Economies”. IMF Working Paper No. 11/51. Consulta: mayo de 2019. 〈http://bit.ly/31n61dU.

Hansen, Niels, y Olga Sulla. 2013. "El crecimiento del crédito en América Latina ¿Desarrollo financiero o boom crediticio?”. Revista Estudios Económicos (25): 51-80. 〈http://bit. ly/31hHZkx>.

Hibbeln, Martin. 2010. Risk Management in Credit Portfolios: Concentration Risk and Basel II. Nueva York: Springer Verlag.

Hicks, Sydney. 1980. "Commercial Banks and Business Loan Behavior". Journal of Banking \& Finance 4 (2): 125-141. Doi:10.1016/0378-4266(80)90002-3.

Hirschman, Albert. 1964. La estrategia del desarrollo económico. Ciudad de México: Fondo de Cultura Económica.

Hirshleifer, David, y Jung Yu. 2012. "Asset Pricing in Production Economies with Extrapolatives Expectations”. Journal of Monetary Economics 76 (1): 87-109. Doi: 10.1016/j.jmon.

Holub, Libor, Michal Nyklíček y Pavel Sedlár. 2014. “Credit Portfolio Sector Concentration and its Implications for Capital Requirements". CNB Financial Stability Report: 131-138. Praga: Czech National Bank.

Imbs, Jean, y Romail Wacziarg. 2002. "Stages of Diversification". The American Economic Review 93 (1): 63-86. 〈http://www.jstor.org/stable/3132162〉.

Jahn, Nayd, Christoph Memmel y Andreas Pfingsten. 2013. 'Banks' Concentration versus Diversification in the Loan Portfolio: New Evidence from Germany". Documento de trabajo No. 53/2013, 12 de diciembre. Consulta: mayo de 2019. 〈https://ideas.repec.org/p/zbw/ bubdps/532013.html.

Jaramillo, Patricio, Sergio Lehmann y David Moreno. 2009. "China, precios de 'commodities' y desempeño de América Latina: algunos hechos estilizados”. Cuadernos de Economía, 46 (133): 67-105. 〈https://bit.ly/2IXhdYf〉. 
King, Robert, y Charles Plosser. 2001. "Money, Credit and Prices in Real Business Cycle". The American Economic Review 74 (3): 363-380. 〈http://bit.ly/2MmYnfl〉.

King, Stephen R. 1986. "Monetary Transmission: Through Bank Loans or Bank Liabilities?”. Journal of Money, Credit and Banking 18 (03): 290-303. Doi: 10.2307/1992382.

Kuodis, Raimondas. 2010. "Makroekonomikos ir finansų sistemos sąveika, burbulai ir krizių ekonomika”. Economics \& Sociology 5 (1): 51-61. 〈http://bit.ly/2tZnUEL〉.

Lanteri, Luis. 2014. "Determinantes de los precios reales del petróleo y su impacto sobre las principales variables macroeconómicas: EU, España, Noruega y Argentina”. Economía: Teoría y Práctica 41 (2): 45-70. 〈https://bit.ly/2VMVs2s〉.

Levine, Ross. 1997. "Financial Development and Economic Growth: Views and Agenda. Journal of Economic Literature 35 (2): 20-45. 〈https://bit.ly/32pUR9u〉.

Lütkebohmert, Eva. 2009. Concentration Risk in Credit Portfolios. Nueva York: Springer.

Martin, Richard, y Tom Wilde. 2002: “Unsystematic Credit Risk". Journal of Risk Magazine 8 (3): 123-128. 〈https://bit.ly/2MPm7rc〉.

Mishkin, Frederic S. 2007. Monetary Policy Strategy. Cambridge: Mit Press.

Mochón, Francisco, y Víctor Beker. 1997. Economía. Principios y aplicaciones. Madrid: Mc Graw-Hill.

Mohanty, Madhusudan, Gert Schnabel y Pablo García. 2006. "Banks and Aggregate Credit: What is New?". En The Banking System in Emergency Economics: How much progress has been made? Madhusudan Montany, editado por Gert Shnabelk y Pablo García, 28-55. Consulta: enero de 2019. 〈https://www.bis.org/publ/bppdf/bispap28b.pdf〉.

Murcia, Andrés, y Hernán Piñeros. 2006. "Determinantes de la oferta de crédito en el sector financiero colombiano". Temas de Estabilidad Financiera 2 (1): 35-51. Doi: 〈https://doi. org/10.32468/tef.21>.

Pazarbasioglu, Ceyla. 2003. "A Credit Crunch? Finland in the Aftermath of the Banking Crisis". Staff Papers 44 (3): 310-325. Doi: 10.2307/3867562.

Pérez, Alicia. 2017. "Elementos aleatorios en espacios normados, propiedades: la ley de los grandes números". Tesis de pregrado, Universidad de Sevilla, Sevilla. 〈https://bit. $1 \mathrm{y} / 321 X O Y J\rangle$.

Portocarrero, Felipe, y Álvaro Tarazona. 2004. “Son rentables las cajas de ahorro y crédito?”. Economía y Sociedad 54 (1): 48-54. 〈https://bit.ly/2OTlZKf〉.

Prebisch, Raúl. 1981. La crisis inflacionaria del capitalismo. Ciudad de México: Fondo de Cultura Económica.

Pyle, David. 1997: “Informational Asymmetries, Financial Structure, and Financial Intermediation". The Journal of Finance 32 (2): 371-387. Doi: 10.2307/2326770.

Rajan, Raghuram, y Luigi Zingales. 1998. "Financial Dependence and Growth". American Economic Review 88 (3): 559-586. 〈https:/www.jstor.org/stable/116849〉.

Rodríguez, Ricardo. 1996. El ejercicio de la banca en México. Ciudad de México: Fondo de Cultura Económica. 


\section{Nicolás Barriga}

Rodríguez, Tomás, y Ligia Gómez. 2002. "Mercado de crédito rural en Nicaragua”. En Crédito para el desarrollo rural en Nicaragua: un enfoque institucional sobre la experiencia del fondo de desarrollo local, editado por Johan Bastiaensen- Nitlapán: Instituto de Investigación y Desarrollo / CLACSO, 55-76.

Sirri, Erick, y Peter Tufano. 1995. The Economics of Pooling. The Global Financial System: A Functional Perspective. Boston: Harvard Business School Press.

Sistemas de Intermediación Financiera Medidos Indiretamente (SIFMI). 2008. Grupo de trabajo sobre cuentas nacionales: estudios estadísticos y prospectivos. Santiago: CEPAL.

Skridulyte, Rita, y Eduardas Freitakas. 2012. "The Measurement of Concentration Risk in Loan Portfolios". Economics \& Sociology 5 (1): 51-61. 〈https://bit.ly/2Mm2OH3〉.

Soss, Neal. 1993. "Real Interest Rates? Get Real! Challenge”. Econ Papers 36 (5): 27-28. 〈http://bit.ly/2NbGCyQ〉.

Steindl, Frank. 2000. "Credit Cards, Economization of Money, and Interest Rates". The Journal of Economic Education 31 (3): 271-279. Doi:10.2307/1183097.

Stiglitz, Joseph, y Andrew Weiss. 1981. "Credit Rationing in Markets with Imperfect Information". The American Economic Review 71 (3): 393-410. «https://www.jstor.org/stable/1802787).

Suárez, Antonio. 1992. Diccionario de economía y finanzas. Madrid: McGraw-Hill.

Tan, Pua. 2012. "Determinants of Credit Growth and Interest Margins in the Philippines and Asia". IMF Working Papers. Consulta: mayo de 2019. 〈https://ssrn.com/abstract $=2127018$ 〉. 〈https://doi.org/10.5089/9781475503524.001〉.

Vasicek, Oldrich. 2002. "Loan Portfolio Value”. Risk Magazine 7 (2): 160-162. Doi: 10.1002/9781119186229.ch19.

Weiss, Leonard. 1958. "A Note on Time Deposit Interest Rates". The Journal of Finance 13 (1): 96-102. Doi:10.2307/2976004.

Wojnilower, Albert. 1985. "Private Credit Demand, Supply, and Crunches-How Different Are the 1980's?”. American Economic Review 75 (2): 351-356. 〈http://bit.ly/2pWBztO〉.

York, Jonathan. 2007. "Bank Concentration Risk". The RMA Journal 4 (2): 52-57. 〈http://bit. ly/3699fFe>. 